Ensayo científico

Los textos publicados son responsabilidad exclusiva de sus autores

\title{
La enseñanza de la matemática y su relación con otras ciencias
}

\section{The teaching of mathematics and its relationship with other sciences}

Recibido 18/10/2021

\author{
Oscar Antonio Campos \\ Doctorado en Educación, Universidad de El \\ Salvador \\ Oscar.campos@ues.edu.sv \\ https://orcid.org/0000-0001-7550-122X
}

Aceptado 16/11/2021

\section{Referencia}

Campos, O. A. (2021). La enseñanza de la matemática y su relación con otras ciencias. Revista Guatemalteca de Educación Superior, 5(1), 127-134.

https://doi.org/10.46954/revistages.v5i1.80

\section{Resumen}

Palabras claves:

matemáticas, disciplina, perseverancia, organización
OBJETIVO: identificar la relación de los métodos de enseñanza de las Matemáticas con otras ciencias y su importancia en sus aplicaciones. MÉTODO: consulta de primeras fuentes en artículos y tesis. RESULTADOS: el estudio de los múltiples usos de las matemáticas en diferentes áreas de la vida económica, cultural y social ayudan a comprender la necesidad de utilizar las matemáticas en beneficio de la sociedad. Además, su aplicación, combinada con los procesos de producción y otras ciencias, favorecen la formación politécnica. CONCLUSIÓN: es importante aclarar que el aprendizaje de las Matemáticas requiere disciplina, perseverancia y organización, entre otros rasgos de personalidad y que además el método de enseñanza de las matemáticas no puede desarrollarse plenamente si no está directamente vinculado a la práctica escolar. 


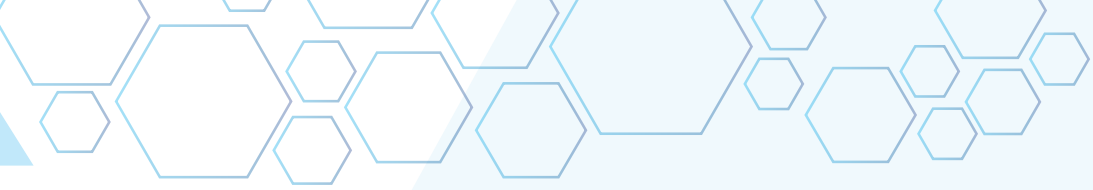

\section{Keywords:}

mathematics, discipline, perseverance, organization.

\section{Abstract}

OBJECTIVE: to identify the relationship of the teaching methods of Mathematics with other sciences and their importance in their applications. METHOD: consultation of first sources in articles and theses. RESULTS: the study of the multiple uses of mathematics in different areas of economic, cultural and social life help to understand the need to use mathematics for the benefit of society. In addition, its application, combined with production processes and other sciences, favor polytechnic training. CONCLUSION: it is important to clarify that learning mathematics requires discipline, perseverance and organization, among other personality traits, and that the teaching method of mathematics cannot be fully developed if it is not directly linked to school practice.

\section{Introducción}

El presente ensayo se propone abordar la relación que tiene la enseñanza de la matemática con otras ciencias, se justifica por la necesidad de conocer el impacto e implicaciones que la enseñanza de la matemática tiene con la psicología. Así mismo su aplicación, combinada con los procesos de producción y otras ciencias, lo cual favorece la formación politécnica, además la concentración y el enfoque en los problemas de aplicación pueden ayudar a promover la conciencia de una producción eficiente y a trabajar por un mundo mejor para todos. El objetivo es de identificar la relación de los métodos de enseñanza de las Matemáticas con otras ciencias y su importancia en sus aplicaciones. Cabe resaltar que este, es producto del capítulo 1 de mi tesis doctoral denominada Estrategia metodológica para la enseñanza de la matemática la cual está dirigida a los profesores del sector público que imparten el curso de matemática en el nivel de educación básica. Cabe considerar por otra parte, que el estudiar los múltiples usos de las matemáticas en diferentes áreas de la vida económica, cultural y social puede ayudar a comprender la necesidad de utilizar las matemáticas en beneficio de la sociedad. 


\section{Contenido}

El aprendizaje de las matemáticas trae consigo muchas posibilidades, lo que contribuye de manera decisiva al desarrollo de muchos aspectos de la personalidad y estimula el perfeccionamiento de diversas habilidades intelectuales como: pensamiento lógico y flexible, imaginación, inteligencia espacial, aritmética mental, creatividad, etc. Así mismo la aplicación de la Matemática juega un importante papel en la planificación de la economía, la dirección de la producción, el diagnóstico y tratamiento de enfermedades, el estudio del rendimiento de atletas, irrumpiendo así todos los campos del saber de la humanidad.

En relación a lo anterior los autores en el artículo Matemática, cotidianidad y pedagogía integral nos mencionan que:

"Un aspecto que debe apoyar el proceso de enseñanza y aprendizaje es la coherencia interna de las matemáticas, que está estrechamente relacionada con la comprensión conceptual, y la capacidad para formular problemas y resolverlos con estrategias. Conectividad con el mundo real o la vida cotidiana y el poder de las matemáticas para modelar problemas de otras áreas de su formación lógica. La conciencia de la importancia de estudiar un mismo concepto en diferentes contextos y establecer relaciones entre diferentes conceptos que contribuyan al tratamiento de un objeto en particular" (Rodríguez, 2010)

Dentro de este marco, existe la opinión de que en el aprendizaje de las matemáticas existen requisitos para la aplicación y el desarrollo de la mente; por ejemplo, realizando inferencias mentales y visualizaciones de relaciones espaciales. La peculiaridad de los objetos matemáticos que son entidades abstractas, así como la lógica de su estructura y la sutileza de su lenguaje, asegura el reconocido respeto por la complejidad de las formas. Por lo tanto, el aprendizaje a menudo requiere disciplina, perseverancia y organización, entre otros rasgos de personalidad. 
Estudiar los múltiples usos de las matemáticas en diferentes áreas de la vida económica, cultural y social puede ayudar a comprender la necesidad de utilizar las matemáticas en beneficio de la sociedad. Su aplicación, combinada con los procesos de producción y otras ciencias, puede favorecer la formación politécnica: la concentración y el enfoque en los problemas de aplicación pueden ayudar a promover la conciencia de una producción eficiente y a trabajar por un mundo mejor para todos.

En la educación matemática se asume que a la hora de resolver un problema siempre hay un cierto tipo de introspección dirigida hacia la vida, y no hacia la evaluación. En el temario de los planes de estudio que el Ministerio de Educación tiene en los cursos de Matemáticas para el tercer ciclo de educación básica, menciona que: "Los docentes deben crear situaciones en el aula en las que los estudiantes aprendan a explorar, aplicar, interpretar y analizar conceptos, ideas, procedimientos, algoritmos u otras disciplinas matemáticas que se suponen deben saber usar" (MINED, 2020)

Por lo que, es necesario advertir que la importancia de la enseñanza de la Matemática en los centros escolares salvadoreños está fundamentada en tres elementos básicos, el razonamiento lógico matemático, la comunicación con lenguaje matemático y por la aplicación de la Matemática al entorno

Ahora que se ha aclarado la importancia de las matemáticas para la sociedad y la importancia de su enseñanza, cabe señalar que la enseñanza de las matemáticas en las escuelas se da como un proceso integrado vinculado al aprendizaje de los estudiantes. Este proceso no se desarrolla automática ni empíricamente, sino con metas bien definidas y según reglas históricamente probadas.

\section{La relación de los métodos de enseñanza de las matemáticas con otras ciencias}

El proceso de enseñanza y aprendizaje debe estar dirigido a que los estudiantes sean sujetos activos en la adquisición de conocimientos, el desarrollo de habilidades y destrezas y el 
manejo de conflictos que deben ser resueltos por su propio aprendizaje. Son precisamente estas contradicciones que surgen en el desarrollo del proceso de enseñanza-aprendizaje en matemáticas las que se convierten en un motor impulsor en el desarrollo de los estudiantes hacia un conocimiento cualitativamente superior.

Los métodos de enseñanza de las matemáticas deben explicar cómo la enseñanza general y los principios didácticos se incorporan a la educación matemática y cómo se aplican los métodos de enseñanza a la asignatura. Por tanto, está muy relacionado con la pedagogía.

El docente debe tener un conocimiento matemático fuerte, pero esto no es suficiente, además, debe amar al alumno, conocer el nivel de conocimientos, destrezas y habilidades del alumno; Investigar las razones que influyen en la eficacia del aprendizaje de las matemáticas; Sobre todo, domine los métodos de enseñanza de las matemáticas. Así mismo, "el docente debe educar a los estudiantes y prepararlos para vivir y crecer en sociedad, desde formarse el concepto de planificar el proceso de enseñanza y aprendizaje de las matemáticas, desde darse cuenta de que los intereses y motivaciones responden a las necesidades de aprendizaje de los estudiantes" (Yoppiz, et al., 2016, p. 5)

En una clase de matemáticas, debemos asegurarnos de cuánta información pueden absorber los estudiantes; la asignación de la carga de trabajo para evitar la fatiga y monotonía; tenga en cuenta a los estudiantes con la visibilidad de la pizarra y la iluminación del aula. Esto facilita la creación de una absorción eficaz, lo cual refleja el vínculo entre la enseñanza de las matemáticas y las ciencias relacionadas con el desarrollo anatómico y fisiológico de niños y adolescentes.

El método de enseñanza de las matemáticas, requiere, entre otras cosas, el conocimiento de la psicología para despertar el interés y la atención de los estudiantes; evitar el olvido y promover la persistencia del conocimiento; incrementar la efectividad 
educativa y desarrollar habilidades y competencias matemáticas; liderar el proceso de abstracción de conocimientos matemáticos en los estudiantes; seleccionar materiales y determinar métodos de enseñanza adecuados.

Un aspecto esencial es que el método de enseñanza de las matemáticas no puede desarrollarse plenamente si no está directamente relacionado con la práctica escolar. Es la fuente de problemas a analizar, en la que se prueban los resultados de las teorías desarrolladas y en la que se encuentran los resultados de sus investigaciones.

De esta forma, se aclaran las relaciones entre la enseñanza de las matemáticas y otras ciencias, y se derivan directamente las relaciones de esta asignatura con otras asignaturas del programa y con el componente de trabajo de la formación del profesorado.

\section{La Matemática y su posición en relación con el resto de las asignaturas del curso escolar}

Para lograr sus objetivos, las matemáticas no están aisladas y no ejercen su influencia de forma independiente. La relación de la educación matemática con otras materias se puede analizar desde tres perspectivas diferentes:

Primero por la aplicación de los conocimiento, hábitos, habilidades y capacidades matemáticas en otras asignaturas. Estos hábitos y habilidades se ven reflejados en el momento de calcular números y cantidades, establecer relaciones entre cantidades, resolver ecuaciones y encontrar aplicaciones metodológicas en la investigación de las ciencias naturales. En ocasiones, en matemáticas, es necesario dar un periodo de tiempo prudencial al procesar algunos conocimientos para facilitar el desarrollo de otros temas. Por tanto, se mejora el conocimiento de los sistemas de coordenadas ortogonales para su uso en la determinación de ubicaciones en mapas geográficos; además, para explicar las fórmulas utilizadas en física y química. 
Segundo por la preparación de los conocimientos, hábitos y habilidades matemáticas mediante la enseñanza de otras asignaturas. Así, la enseñanza de otras materias es una parte activa del trabajo preparatorio para adquirir conocimientos y desarrollar habilidades y hábitos matemáticos. Este es el caso de la física donde, por ejemplo, se utilizan conocimientos previos de operaciones vectoriales, que luego se utilizan en matemáticas para estudiar ecuaciones paramétricas de líneas rectas.

Y tercero por la relación entre el contenido de la enseñanza de la Matemática y el de otras asignaturas. Desde este punto de vista, la relación entre matemáticas y otras asignaturas se puede establecer mediante, la resolución de problemas de aplicación, la motivación para abordar determinadas unidades temáticas y la contribución de cada asignatura a la educación ideológica, política y al desarrollo intelectual de los estudiantes

\section{Conclusión}

De todo lo anterior, podemos entender que un aspecto fundamental es que el método de enseñanza de las matemáticas no puede desarrollarse plenamente si no está directamente vinculado a la práctica escolar, cabe considerar por otra parte que la enseñanza de otras materias es una parte activa del trabajo preparatorio para adquirir conocimientos y desarrollar habilidades y hábitos matemáticos y que además, la relación de la enseñanza de la matemática con otras materias se puede analizar desde distintos ángulos o perspectivas.

\section{Referencias bibliográficas}

MINED (2020). Programa de estudios de tercer ciclo de educación Básica, Sitio WEB:https://www.mined.gob.sv/ download/programa-de-estudio-de-tercer-ciclo/

Rodríguez, M. E. (2010). Matemática, cotidianidad y pedagogía integral: tendencias oferentes desde una óptica humanista integral. REIFOP, 13(3), 105-112. http//www. aufop.com 
Vásquez Rodríguez, F. (2010). Estrategias de enseñanza:

investigaciones sobre didáctica en instituciones

educativas de la ciudad de Pasto. Compilador. Bogotá, Colombia. http://biblioteca.clacso.edu.ar/Colombia/fceunisalle/20170117011106/Estrategias.pdf

Yoppiz, Y., Cruz, A., Gamboa, M. E. y Osorio, G. (2016). Alternativa didáctica para contribuir al perfeccionamiento de la planificación del proceso de enseñanza-aprendizaje de la Matemática en la carrera Licenciatura en Educación Matemática-Física. Boletín Redipe, 5(5). 147-164. https:// dialnet.unirioja.es/servlet/articulo?codigo $=6064411$

\section{Sobre el autor}

Docente a tiempo completo con 18 años de experiencia laboral en la Universidad de El Salvador, Graduado de Licenciado en Matemática por la Universidad de El Salvador con Maestría en Profesionalización de la Docencia Superior, Universidad de El Salvador

Maestría en Técnicas de Investigación Social, Universidad de El Salvador y Candidato a Doctor en Educación con Especialidad en Educación Básica, Universidad de El Salvador

\section{Conflicto de intereses}

Declara no tener ningún conflicto de intereses.

\section{Declaración de consentimiento informado}

El estudio se realizó con fines educativos, respetando el Código de ética y buenas prácticas editoriales de publicación.

\section{Derechos de uso Copyright@ 2021 por Oscar Antonio Campos \\ Este texto está protegido por la Licencia Creative Commons Atribución 4.0 Internacional.}

Usted es libre para compartir, copiar y redistribuir el material en cualquier medio o formato y adaptar el documento, remezclar, transformar y crear a partir del material para cualquier propósito, incluso comercialmente, siempre que cumpla la condición de atribución: usted debe reconocer el crédito de una obra de manera adecuada, proporcionar un enlace a la licencia, e indicar si se han realizado cambios. Puede hacerlo en cualquier forma razonable, pero no de forma tal que sugiera que tiene el apoyo del licenciante o lo recibe por el uso que hace. 